\title{
Two Absolute Index-Summability Methods
}

\author{
${ }^{1}$ S. Sarangi, ${ }^{2}$ S.K. Paikray ${ }^{*},{ }^{3}$ M. Dash, ${ }^{4}$ M. Misra, ${ }^{5}$ U.K. Misra \\ ${ }^{1}$ Research scholar Department of Mathematics, \\ Ravenshaw University, Cuttack, Odisha, India, \\ sunitas1970@gmail.com \\ ${ }^{2}$ Department of Mathematics, \\ VSSUT, Burla, Odisha, India \\ skpaikray_math@vssut.ac.in \\ ${ }^{3}$ Department of Mathematics, \\ Ravenshaw University, Cuttack, Odisha, India, \\ dashminakshi@gmail.com \\ ${ }^{4}$ Department of Mathematics, \\ B.A. College, Berhampur, Odisha, India \\ mehendramisra2007@gmail.com \\ ${ }^{5}$ Department of Mathematics \\ NIST, Berhampur, Odisha, India \\ umakanta_misra@yahoo.com
}

\section{Abstract}

In this paper we have established a relation between the Summability methods $X-\left|\bar{N}, p_{n}\right|_{k}$ and $Y-|A, \delta, \gamma|_{k}, k \geq 1, \delta \geq 0$.

Keywords: $X-\left|\bar{N}, p_{n}\right|_{k}, X-|A|_{k}, Y-|A, \delta|_{k}, Y-|A, \delta, \gamma|_{k}, k \geq 1, \delta \geq 0$ - summabilities.

Ams Classification No: 40D25

\section{Introduction :}

Let $\sum a_{n}$ be an infinite series and $\left\{s_{n}\right\}$ the sequence of partial sums. Let $\left\{p_{n}\right\}$ be a sequence of non-negative numbers with $P_{n}=\sum_{v=0}^{n} p_{v}$ for all $n \in N$. The sequence-to-sequence transformation

$$
t_{n}=\frac{1}{P_{n}} \sum_{v=0}^{n} p_{v} s_{v}, P_{n} \neq 0
$$

dfines $\left|\bar{N}, p_{n}\right|$-mean of the sequence $\left\{s_{n}\right\}$ generated by the sequence of coefficients $\left\{p_{n}\right\}$. The series $\sum a_{n}$ is said to be summable $\left|\bar{N}, p_{n}\right|_{k}, k \geq 1$, [4] if

$$
\sum_{n=1}^{\infty}\left(\frac{P_{n}}{p_{n}}\right)^{k-1}\left|t_{n}-t_{n-1}\right|^{k}<\infty
$$

The sequence -to-sequence transformation

$$
\tau_{n}=\frac{1}{P_{n}} \sum_{v=0}^{n} p_{n-v} s_{v}, P_{n} \neq 0
$$

defines $\left|N, p_{n}\right|$-mean of the sequence $\left\{s_{n}\right\}$. The series $\sum a_{n}$ is said to be summable $\left|N, p_{n}\right|_{k}, k \geq 1$, if Corresponding Author: skpaikray_math@vssut.ac.in

$$
\sum_{n=1}^{\infty}\left(\frac{P_{n}}{p_{n}}\right)^{k-1}\left|\tau_{n}-\tau_{n-1}\right|^{k}<\infty .
$$

The series $\sum a_{n}$ is said to be summable $X-\left|\bar{N}, p_{n}\right|_{k}, k \geq 1$, if 


$$
\sum_{n=1}^{\infty} X_{n}^{k-1}\left|t_{n}-t_{n-1}\right|^{k}<\infty
$$

where $\left\{X_{n}\right\}$ is a sequence of positive real constants. Similarly, $\sum a_{n}$ is said to be summable $X-\left|N, p_{n}\right|_{k}, k \geq 1$, if

$$
\sum_{n=1}^{\infty} X_{n}^{k-1}\left|\tau_{n}-\tau_{n-1}\right|^{k}<\infty
$$

Let $A=\left(a_{n k}\right)$ be a $\infty \times \infty$ matrix. The series $\sum a_{n}$ is said to be summable $X-|A|_{k}, k \geq 1$, if

$$
\sum_{n=1}^{\infty} X_{n}^{k-1}\left|T_{n}-T_{n-1}\right|^{k}<\infty,
$$

$\sum a_{n}$ is said to be summable $X-|A, \delta|_{k}, k \geq 1, \delta \geq 0$, if

$$
\sum_{n=1}^{\infty} X_{n}^{\delta k+k-1}\left|T_{n}-T_{n-1}\right|^{k}<\infty
$$

and for any real number $\gamma, \sum a_{n}$ is said to be summable $X-|A, \delta, \gamma|_{k}, k \geq 1, \delta \geq 0$, if

$$
\sum_{n=1}^{\infty} X_{n}^{\gamma(\delta k+k-1)}\left|T_{n}-T_{n-1}\right|^{k}<\infty
$$

where the sequence -to-sequence transformation $\left\{T_{n}\right\}$ is given by

$$
T_{n}=\sum_{k=1}^{\infty} a_{n k} s_{k}
$$

\section{Known Theorems:}

\section{Theorem-A[1]:}

Dealing with the index summability method Bor has established the following theorems:

$$
\begin{aligned}
& \text { Let }\left\{p_{n}\right\} \text { be a sequence of positive real constants such that as } n \rightarrow \infty \\
& \begin{array}{ll}
\text { i) } n p_{n}=O\left(P_{n}\right) & \text { ii) } P_{n}=O\left(n p_{n}\right) \text {. }
\end{array}
\end{aligned}
$$

If $\sum a_{n}$ is summable $|C, 1|_{k}$ then it is summable $\left|\bar{N}, p_{n}\right|_{k}, k \geq 1$.

\section{Theorem-B [2]:}

Let $\left\{p_{n}\right\}$ be a sequence of positive real constants such that as $n \rightarrow \infty$
(i) $n p_{n}=O\left(P_{n}\right)$
(ii) $P_{n}=O\left(n p_{n}\right)$.

If $\sum a_{n}$ is summable $\left|\bar{N}, p_{n}\right|_{k}$ then it is summable $|C, 1|_{k}, k \geq 1$.

Subsequently Bor and Thorpe established the following result.

\section{Theorem-C [3]:}

Let $\left\{p_{n}\right\}$ and $\left\{q_{n}\right\}$ be the sequence of positive real constants such that

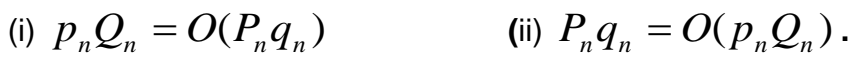

then the series $\sum a_{n}$ is summable $\left|\bar{N}, q_{n}\right|_{k}$ whenever it is summable $\left|\bar{N}, p_{n}\right|_{k}, k \geq 1$.

Further , Tripathi established

\section{Theorem-D [5]:}

Suppose $\left\{p_{n}\right\},\left\{q_{n}\right\},\left\{X_{n}\right\}$ and $\left\{Y_{n}\right\}$ are sequences of positive real constants such that
(i) $q_{n} p_{n}=O\left(p_{n} q_{n}\right)$
(ii) $Q_{n}=O\left(q_{n} X_{n}\right)$
(iii) $Y_{n} p_{n}=O\left(p_{n}\right)$.

If $\sum a_{n}$ is summable $X-\left|\bar{N}, p_{n}\right|_{k}$, then it is summable $Y-\left|\bar{N}, q_{n}\right|_{k}, k \geq 1$.

Extending the above result , Misra, Misra and Routa established the following theorem replacing $Y-\left|\bar{N}, q_{n}\right|_{k}, k \geq 1$ by $Y-\left|N, q_{n}\right|_{k}, k \geq 1$, in Theorem-D.

\section{Theorem-E [6]:}


Suppose $\left\{p_{n}\right\},\left\{q_{n}\right\},\left\{X_{n}\right\}$ and $\left\{Y_{n}\right\}$ are sequences of positive real constants such that
(i) $q_{n} p_{n}=O\left(p_{n} q_{n}\right)$
(ii) $Q_{n}=O\left(q_{n} X_{n}\right)$
(iii) $Y_{n} p_{n}=O\left(p_{n}\right)$
(iv) $\sum_{n=v+1}^{n+1} \frac{1}{Q_{n}}=O\left(\frac{1}{Q_{\gamma}}\right)$
(v) $\sum_{v=1}^{n-1} a_{v}^{\frac{k}{k-1}}=O(1), k \neq 1$.

If $\sum a_{n}$ is summable $X-\left|\bar{N}, p_{n}\right|_{k}$, then it is summable $Y-\left|N, q_{n}\right|_{k}, k \geq 1$.

Subsequently Misra et al established the following theorem:

Theorem-F [7] :

Suppose $\left\{p_{n}\right\},\left\{q_{n}\right\},\left\{X_{n}\right\}$ and $\left\{Y_{n}\right\}$ are sequences of positive real constants such that
( i) $a_{n n} P_{n}=O\left(p_{n}\right)$
(ii) $\frac{1}{a_{n n}}=O\left(X_{n}\right)$,
(iii) $Y_{n} p_{n}=O\left(P_{n}\right)$,
(iv) $\sum_{n=r}^{m+1}\left(\frac{P_{n}}{p_{n}}\right)^{\delta k+k-1} A_{n r}=O\left(a_{r r}\right)$, where $A_{n k}=\sum_{v=k}^{n} a_{n v}$
(v) $\sum_{r=1}^{n} A_{n r}=O(1)$.

and

Then $\sum a_{n}$ is $Y-|A, \delta|_{k}, k \geq 1, \delta \geq 0$ summable whenever $\sum a_{n}$ is summable $X-\left|\bar{N}, p_{n}\right|_{k}, k \geq 1$.

In what follows, in the present paper we generalize the above theorem the matrix Summability.

\section{Main Theorem:}

Suppose $\left\{p_{n}\right\},\left\{q_{n}\right\},\left\{X_{n}\right\}$ and $\left\{Y_{n}\right\}$ are sequences of positive real constants such that

$$
a_{n n} P_{n}=O\left(p_{n}\right) \text {, }
$$

$$
\frac{1}{a_{n n}}=O\left(X_{n}\right)
$$

and

$$
Y_{n} p_{n}=O\left(P_{n}\right) \text {, }
$$

$$
\sum_{n=r}^{m+1}\left(\frac{P_{n}}{p_{n}}\right)^{\gamma(\delta k+k-1)} A_{n r}=O\left(a_{r r}\right) \text {, where } A_{n k}=\sum_{v=k}^{n} a_{n v}
$$

(3)

$$
\sum_{r=1}^{n} A_{n r}=O(1)
$$

Then $\sum a_{n}$ is $Y-|A, \delta|_{k}, k \geq 1, \delta \geq 0$ summable whenever $\sum a_{n}$ is summable $X-\left|\bar{N}, p_{n}\right|_{k}, k \geq 1$.

\section{Proof of the Theorem:}

If $\left\{t_{n}\right\}$ is the nth $\left|\bar{N}, p_{n}\right|$-mean of $\sum a_{n}$, then

$$
\begin{aligned}
t_{n} & =\frac{1}{P_{n}} \sum_{v=0}^{n} p_{v} s_{v} \\
& =\frac{1}{P_{n}}\left\{p_{0} a_{0}+p_{1}\left(a_{0}+a_{1}\right)+\cdots+p_{n}\left(a_{0}+a_{1}+\cdots+a_{n}\right)\right\} \\
& =\frac{1}{P_{n}}\left\{P_{n} a_{0}+\left(P_{n}-p_{0}\right) a_{1}+\cdots+\left(P_{n}-p_{n-1}\right) a_{n}\right\} \\
& =\frac{1}{P_{n}} \sum_{v=0}^{n}\left(P_{n}-P_{v-1}\right) a_{v}
\end{aligned}
$$


Then

$$
\begin{aligned}
\Delta t_{n} & =t_{n}-t_{n-1} \\
& =\frac{1}{P_{n}} \sum_{v=0}^{n}\left(P_{n}-P_{v-1}\right) a_{v}-\frac{1}{P_{n-1}} \sum_{v=0}^{n-1}\left(P_{n-1}-P_{v-1}\right) a_{v} \\
& =\frac{1}{P_{n}} \sum_{v=1}^{n}\left(P_{n}-P_{v-1}\right) a_{v}-\frac{1}{P_{n-1}} \sum_{v=0}^{n-1}\left(P_{n-1}-P_{v-1}\right) a_{v} \\
& =\left(\frac{1}{P_{n-1}}-\frac{1}{P_{n}}\right) \sum_{v=1}^{n} P_{v-1} a_{v} \\
& =\frac{p_{n}}{P_{n} P_{n-1}} \sum_{v=1}^{n} P_{v-1} a_{v}
\end{aligned}
$$

Hence,

$$
\frac{P_{n} P_{n-1}}{p_{n}} \Delta t_{n}=\sum_{v=1}^{n} P_{v-1} a_{v}
$$

and

$$
\frac{P_{n-1} P_{n-1}}{p_{n-1}} \Delta t_{n-1}=\sum_{v=1}^{n-1} P_{v-1} a_{v}
$$

Thus,

$$
a_{n}=\frac{P_{n}}{p_{n}} \Delta t_{n}-\frac{P_{n-2}}{p_{n-1}} \Delta t_{n-1}
$$

Further, if $\left\{T_{n}\right\}$ is the nth $A$-mean of $\sum a_{n}$, where $A=\left(a_{n k}\right)_{\infty x \times \infty}$ triangular matrix,

Then,

$$
\begin{aligned}
T_{n} & =\sum_{k=0}^{n} a_{n k} s_{k} \\
& =\sum_{k=0}^{n} A_{n k} a_{k}, A_{n k}=\sum_{v=k}^{n} a_{n v}
\end{aligned}
$$

Now,

$$
\begin{aligned}
T_{n}-T_{n-1} & =\sum_{k=0}^{n} A_{n k} a_{k}-\sum_{k=0}^{n-1} A_{n-1, k} a_{k} \\
& =\sum_{k=1}^{n}\left(A_{n k}-A_{n-1, k}\right) a_{k} \\
& =\sum_{k=1}^{n}\left(A_{n k}-A_{n-1, k}\right)\left(\frac{P_{k}}{p_{k}} \Delta t_{k}-\frac{P_{k-2}}{p_{k-1}} \Delta t_{k-1}\right) \\
=\sum_{k=1}^{n} A_{n k} \frac{P_{k}}{p_{k}} \Delta t_{k} & -\sum_{k=1}^{n} A_{n k} \frac{P_{k-2}}{p_{k-1}} \Delta t_{k-1}-\sum_{k=1}^{n-1} A_{n-1, k} \frac{P_{k}}{p_{k}} \Delta t_{k}+\sum_{k=1}^{n-1} A_{n-1, k} \frac{P_{k-2}}{p_{k-1}} \Delta t_{k-1} \\
& =S_{1}+S_{2}+S_{3}+S_{4} \text { (say). }
\end{aligned}
$$

$$
\sum_{n=1}^{m+1} Y_{n}^{\delta k+k-1}\left|T_{n}-T_{n-1}\right|^{k} \leq \sum_{n=1}^{m+1} Y_{n}^{\gamma(\delta k+k-1)}\left|S_{1}+S_{2}+S_{3}+S_{4}\right|^{k}=\sum_{i=1}^{4} \sum_{n=1}^{m+1} Y_{n}^{\gamma(\delta k+k-1)}\left|S_{i}\right|^{k}
$$

(By Minokowski's inequality)

Our Theorem will be established if we show that $\sum_{n=1}^{m+1} Y_{n}^{\delta k+k-11}\left|S_{i}\right|^{k}<\infty, \forall i=1,2,3,4$.

$$
\sum_{n=1}^{m+1} Y_{n}^{\gamma(\delta k+k-1)}\left|S_{1}\right|^{k}=\sum_{n=1}^{m+1} Y_{n}^{\gamma(\delta k+k-1)}\left|\sum_{r=1}^{n} A_{n r} \frac{P_{r}}{p_{r}} \Delta t_{r}\right|^{k}
$$




$$
\begin{aligned}
& \leq \sum_{n=1}^{m+1} Y_{n}^{\gamma(\delta k+k-1)} \sum_{r=1}^{n}\left(\frac{P_{r}}{p_{r}}\right)^{k}\left|\Delta t_{r}\right|^{k} A_{n r}\left(\sum_{r=1}^{n} A_{n r}\right)^{k-1} \\
& =O(1) \sum_{r=1}^{m+1}\left(\frac{P_{r}}{p_{r}}\right)^{k}\left|\Delta t_{r}\right|^{k} \sum_{n=r}^{m+1} Y_{n}^{\gamma(\delta k+k-1)} A_{n r}, \text { by (3.5) } \\
& =O(1) \sum_{r=1}^{m+1}\left(\frac{P_{r}}{p_{r}}\right)^{k}\left|\Delta t_{r}\right|^{k} \sum_{n=r}^{m+1}\left(\frac{P_{n}}{p_{n}}\right)^{\gamma(\delta k+k-1)} A_{n r}, \text { by (3.3) } \\
& =O(1) \sum_{r=1}^{m+1}\left(\frac{1}{a_{r r}}\right)^{k}\left|\Delta t_{r}\right|^{k} a_{r r}, \text { (using 3.4) } \\
& =O(1) \sum_{r=1}^{m+1} X_{r}^{k-1}\left|\Delta t_{r}\right|^{k}, \text { (using 3.2) } \\
& =O(1) .
\end{aligned}
$$

Next,

Also,

$$
\begin{aligned}
\sum_{n=1}^{m+1} Y_{n}^{\gamma(\delta k+k-1)}\left|S_{3}\right|^{k} & =\sum_{n=1}^{m+1} Y_{n}^{\gamma(\delta k+k-1)}\left|\sum_{r=1}^{n=1} A_{n-1, r} \frac{P_{r}}{p_{r}} \Delta t_{r}\right|^{k} \\
& \leq \sum_{n=1}^{m+1} Y_{n}^{\gamma(\delta k+k-1)} \sum_{r=1}^{n-1}\left(\frac{P_{r}}{p_{r}}\right)^{k}\left|\Delta t_{r}\right|^{k} A_{n-1, r}\left(\sum_{r=1}^{n} A_{n-1, r}\right)^{k-1} \\
& =O(1) \sum_{r=1}^{m+1}\left(\frac{P_{r}}{p_{r}}\right)^{k}\left|\Delta t_{r}\right|^{k} \sum_{n=r+1}^{m+1}\left(\frac{P_{n}}{p_{n}}\right)^{\gamma(\delta k+k-1)} A_{n-1, r}, \text { using (3.5) } \\
& =O(1) \sum_{r=1}^{m+1}\left(\frac{1}{a_{r r}}\right)^{k}\left|\Delta t_{r}\right|^{k} a_{r r}, \text { (using 3.4) }
\end{aligned}
$$


Finally,

$$
\begin{aligned}
& =O(1) \sum_{r=1}^{m+1} X_{r}^{k-1}\left|\Delta t_{r}\right|^{k}, \text { (using 3.2) } \\
& =O(1)
\end{aligned}
$$

$$
\begin{aligned}
\sum_{n=1}^{m+1} Y_{n}^{\gamma(\delta k+k-1)}\left|S_{4}\right|^{k}= & \sum_{n=1}^{m+1} Y_{n}^{\gamma(\delta k+k-1)}\left|\sum_{r=1}^{n=1} A_{n-1, r} \frac{P_{r-2}}{p_{r-2}} \Delta t_{r-1}\right|^{k} \\
& \leq \sum_{n=1}^{m+1} Y_{n}^{\gamma(\delta k+k-1)} \sum_{r=1}^{n-1}\left(\frac{P_{r-2}}{p_{r-2}}\right)^{k}\left|\Delta t_{r-1}\right|^{k} A_{n-1, r}\left(\sum_{r=1}^{n} A_{n-1, r}\right)^{k-1} \\
& =O(1) \sum_{n=1}^{m+1} Y_{n}^{\gamma(\delta k+k-1)} \sum_{r=1}^{n-1}\left(\frac{P_{r-1}}{p_{r-1}}\right)^{k}\left|\Delta t_{r-1}\right|^{k} A_{n-1, r} \\
& =O(1) \sum_{r=1}^{m}\left(\frac{P_{r-1}}{p_{r-1}}\right)^{k}\left|\Delta t_{r-1}\right|^{k} \sum_{n=r+1}^{m+1} A_{n-1, r} Y_{n}^{\gamma(\delta k+k-1)} \\
& =O(1) \sum_{r=1}^{m}\left(\frac{1}{a_{r-1, r-1}}\right)^{k}\left|\Delta t_{r-1}\right|^{k} a_{r-1, r-1}, \text { (using 3.4) } \\
& =O(1) \sum_{r=1}^{m} X_{r-1}^{k-1}\left|\Delta t_{r-1}\right|^{k},(\text { using 3.2) } \\
& =O(1) .
\end{aligned}
$$

\section{References}

1. Bor. H, A note On two Summability methods", Math.Proc. Camb. Phil.Soc.,Vol.97(1985),147-149.

2. Bor. H, "A note On two Summability methods", Proc.Amer. Math. Soc.,Vol.98(1986),81-84.

3. Bor. H, Thorpe, "On some Summability methods",Analysis, Vol.7(1987),Pp-147-152.

4. Mazher ,S.M ., "Absolute Summability factor for Fourier Series” ,Indian J.Math., Vol.41,No.2(1999),221-229.

5. Tripathy ,Sanjaya, "A Relation between two Summability Methods", Bulletin of Pure and Applied Sciences.Vol.20E, No.1 (2001).

6. Misra ,U.K ., Misra ,M. and Rauto, K., "Relation between two Summability Methods" ,Acta Ciencia Indica,Vol.XXXI M, No.4 (2005), 1173-1179

7. Misra ,U.K ., Misra ,M., Padhy ,B.P. and Panda ,N.K."On Relation between two Absolute Index-Summability Methods" , Acta Ciencia Indica,Vol.XXXI M, No.4 (2005), 1173-1179 